\title{
The role of C-terminal-pro-endothelin-1 in predicting ischemic stroke outcome
}

\author{
Laura Westphal ${ }^{1}$, Felix Fluri², Mirjam Christ-Crain ${ }^{3}$, Juliane Schweizer ${ }^{1}$, Andreas Luft ${ }^{1}$, Mira Katan $^{1}$
}

1 Department of Neurology, University Hospital Zürich, Zürich, Switzerland; 2 Department of Neurology, University Hospital Wuerzburg, Germany; 3 Department of Endocrinology, Diabetology and Metabolism, University Hospital Basel, Switzerland.

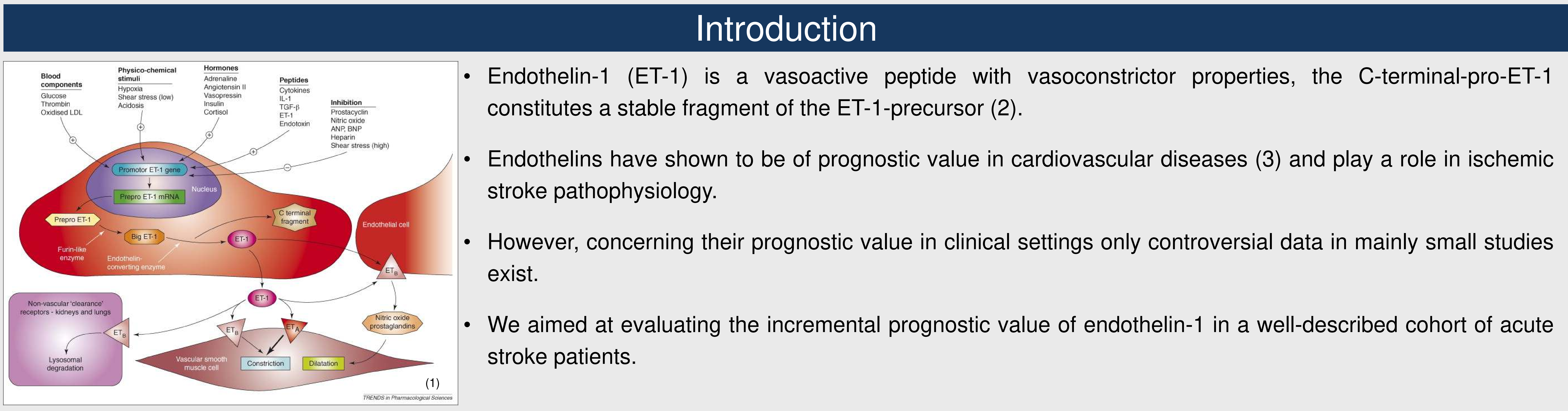

\section{Methods}

- In this prospective cohort-study we prospectively measured endothelin-1 levels of 359 ischemic stroke patients within 72 hours of symptom onset.

- Outcome measures were functional outcome (defined as a modified Rankin Scale of $\leq 2$ for good and $\geq 3$ for unfavourable outcome) and mortality assessed after 90 days.

- We calculated logistic regression and cox proportional hazards models, respectively, to estimate the association of endothelin-1 with our outcome measures after adjusting for demographic and medical risk factors.

- To assess the discriminatory accuracy and the incremental value of endothelin-1 beyond known risk factors, we calculated and compared the area under the receiver operating characteristics (AUC) curve.

- To further estimate the additive benefit of ET-1 to the traditional outcome predictors, we calculated the net reclassification improvement (NRI).

\section{Results}

In the univariate analysis endothelin-1 was associated with unfavourable outcome with an $\mathrm{OR}$ of 1.32 [95\% $\mathrm{Cl}, 1.16-1.51]$ and with mortality with a $\mathrm{HR}$ of 1.45 [95\% Cl, $1.29-1.63]$. After adjusting, endothelin-1 remained in the multivariate analysis an independent predictor for mortality with an adjusted $\mathrm{HR}$ of 1.58 [95\% Cl, $1.34-1.85]$, but not for functional outcome. Adding endothelin-1 to the cox-regression model for mortality, the discriminatory accuracy improved from $0.89[95 \% \mathrm{Cl}, 0.84-0.94]$ to $0.92[95 \% \mathrm{Cl}, 0.88-0.96] \mathrm{p}<0.001$. The addition of $\mathrm{ET}-1$ to the multivariate model for mortality led to a continous $\mathrm{NRI}$ of 0.76 [95\% Cl, $0.32-1.15]$.
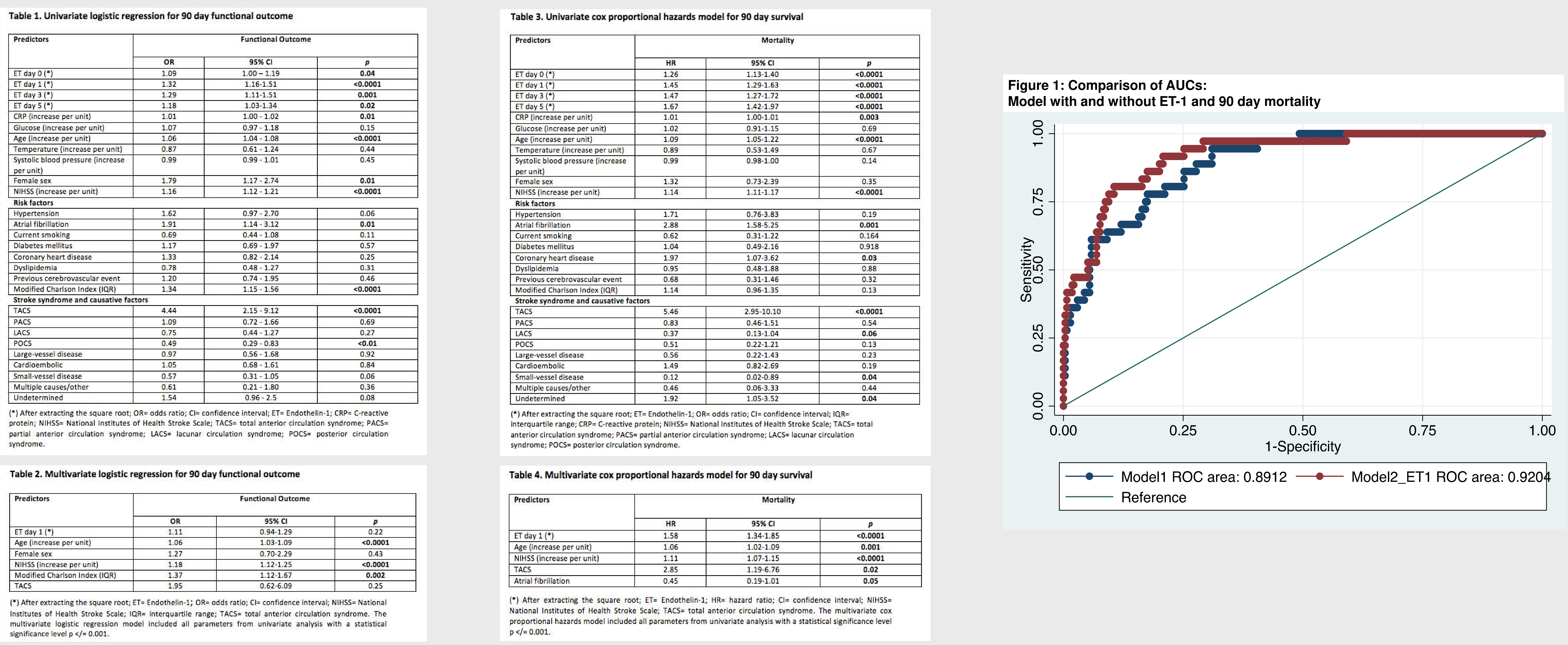

\section{Conclusion}

Endothelin-1 is an independent predictor of mortality in ischemic stroke adding incremental prognostic value beyond traditional demographic and vascular risk factors. These results need further validation in independent larger cohorts.

References:

Dhaun N, Pollock DM, Goddard J and Webb D. Selective and mixed endothelin receptor antagonism in cardiovascular disease. Trends Pharmacol Sci. 2007 Nov;28(11):573-9.
Habib Aet al. Plasma C-terminal pro-endothelin-1 is associated with target-organ damage in African Ammericans with hypertension. Am J Hypertens. 2010 Nov 23(11):1204-8. 